\title{
On the Impact of Monitoring Router Energy Consumption for Greening the Internet
}

\author{
A. Adelin, P. Owezarski, and T. Gayraud \\ CNRS ; LAAS ; 7, avenue du Colonel Roche, F-31077 Toulouse, France \\ Université de Toulouse ; UPS ; INSA, INP, ISAE ; LAAS ; F-31077 Toulouse, France \\ \{aadelin, owe, gayraud\}@laas.fr
}

\begin{abstract}
Research in the field of green-networking is raising more and more interest, in particular driven by energy saving purposes. The global Internet and its thousands of equipments consume an enormous energy amount, have an impact on global warming, and up to now, nobody really addresses subjects dealing with reducing its negative impact on the environment. In addition, nobody has a precise idea about what the Internet - or at least one of its AS (Autonomous System) - consumes. It is obvious that for designing new routing or management strategies for greening the Internet relies on an initial study of the energy consumption of network equipments at large, and routers on a more focuses way. That is why we study in this paper the power consumption of a router depending on several factors as the traffic rate it has to compute, and its configuration (in particular depending on queue management policy). This work then aims to establish an effective method to measure and analyze the power consumption of a router, as well as to provide data from a real router. This work was motivated by the fact that very little data on the power consumption of network devices is available, despite its huge importance for greening network communication. Based on these first results, a discussion is started on how it would be possible to change routing and management strategies and policies in the Internet for saving energy.
\end{abstract}

Index Terms - Electrical power consumption, Router, Wattmeter, energy saving, Internet greening, Green Internet management

\section{INTRODUCTION}

$\mathrm{N}$ owadays, global warming is a problem that is becoming increasingly worrying. According to numerous studies, it is undeniable that the human impact on this phenomenon is indisputable. It is therefore essential to reduce our impact on the environment. Many efforts are being made in many areas, and ICT makes no exception. A recent study [1] has shown that the impact of ICT is estimated between $2 \%$ and $10 \%$ of global energy consumption. A 2008 study for the Department of Ecology in France [2] indicated that the power consumption of ICT in France was 58.6 TWh / year, which represents $13.5 \%$ of the total French power consumption. This power consumption consists of the consumption of data centers (4 TWh), ISPs networks, and private corporate networks (20 TWh), workstations (18 TWh), Televisions and VCRs (16.5 TWh) and phones (0.1 TWh).

This study also refers to the difficulty of measuring the power consumption of ISPs as well as private corporate networks. However it is estimated that the global Internet consumes 16 times more than the consumption of the Paris city, the hourly power consumption of a character from Second Life is equivalent to that of a Brazilian individual. This consumption is due to both equipments for the transmission of information and data centers that store information.

This paper addresses the power consumption of a router depending on its configuration (especially queue management policy) and traffic it has to compute. For this purpose, it first aims to put forward an effective method for measuring the consumption of a router, but also for providing new evidence on consumption characteristics. Based on these first results, it also aims at discussing how the Internet management could be changed for saving energy. The rest of this paper is organized as follows: Section II briefly describes the known work in the field of green-networking. Section III presents the electrical power consumption measurement equipment at our disposal, and the method we designed for getting significant and meaningful electrical power consumption measurement results. Then, section IV details the experiments that we conducted, including the impact of traffic levels, and of the introduction of QoS and BGP announcements on router consumption. These results are interpreted at the end of the section. Then, section $\mathrm{V}$ discusses about possible strategies for managing routing and traffic engineering in order to save energy. The proposal relies obviously on the first results got when measuring router energetic consumption. Section VI concludes the paper.

\section{RELATED WORK}

The energy saving has become a subject of growing importance in the network field. Thus, since very recently, many research teams around the world started working on the green-networking. In 2003, in their pioneering work, Maruti Gupta and Suresh Singh [3] have identified the research topics in green networking which are studied today. Many teams are working on routers, switches and network cards to get some energy efficiency. This work is in particular conducted by the Ethernet Alliance and IEEE 802.3 Energy Efficient Ethernet Study Group. They study the possibility of adapting the transmission rate depending on the bandwidth used: this method is called Adaptive Link Rate (ALR) [5] [6]. According to these studies, a $2 \mathrm{~W}$ economy can be achieved on a router by simply passing from an interface speed of $1 \mathrm{~Gb}$ to $100 \mathrm{Mb}$. A similar change on switches leads to an economy of 
4W. In 2006, 50\% of desktop computers can use the ALR. The related saving is estimated around \$80 millions However this technique is not yet implemented in network equipments.

Another technique called proxying holds our attention, and is studied in [4], [5] and [7]. This technique aims to make a network equipment sleep and to transfer the management of responses to requests from the network to a specific entity. We believe that this opportunity should be followed. There are many terminal devices which do not need to operate constantly. However as we shall see in section V, it would be difficult to apply this technique to routers which are a very strategic network device.

Another topic of research aims to achieve energy savings by designing protocols that take into account the network load, as in [9] and [10] where authors put forward the possibility of turning off unused Ethernet interfaces of switches, or as in [8], based on an evaluation of redundant resources, stopping a part of the network is proposed. These two studies become more meaningful when comparing them to the study presented in [12]. In this study different energy profiles are presented. These profiles depend on the capability of devices to include energy efficiency constraints. Based on these profiles, they determine the energy savings possible on a network.

The last study we want to speak about here describes a similar approach compared to the one proposed in this paper [13]. This study is very interesting because it is based on a concrete study of the power consumption of two routers. The measured data are used to develop a consumption model. This model is then used to determine the potential energy savings when routing traffic. This study has some similarities with the one proposed in this paper. Both studies aim to complement the data collected.

In next section, before turning to experimental results, we will discuss the method we have developed and described the material used.

\section{MEASUREMENT METHOD AND MATERIALS}

Before getting into the experimental platform details, the paper first clarifies what has been studied. The goal is to measure the electrical power consumed (in watts) by a router depending on the traffic it has to compute and its configuration. The extent of this power requires first to know how the router is powered, i.e. whether it is powered by direct current (DC) or alternating current (AC). This issue is very important since it will determine the equipment to be used to measure this power.

\section{A. Theoretical Background}

When the router is powered by a DC, the power consumption is the multiplication of the Root Mean Square (RMS) voltage by the RMS current:

$$
P=U \times I
$$

Thus, as the voltage is fixed, it is enough to measure the current value to obtain the power. However if the router is powered by an alternating current, the power calculation involves an additional term:

$$
P=U \times I \times \cos (\phi)
$$

Where $\varnothing$ is the phase difference between current and voltage. In this case it is necessary to measure both the current value and the phase shift value.

\section{B. Two Practical Approaches}

Two solutions are commonly used: the first consists in using a current clamp. This clamp uses a measurement of the magnetic field created by the passage of current in the conductor for estimating the value of this current. The clamp should only be placed around the current carrying cable, i.e. the cable phase. This solution is particularly suitable for DC current measurement but cannot be used on AC source since the value of the phase shift cannot be obtained this way.

The other method deals with using a wattmeter that measures both voltage and current to determine an exact value of the power consumed. This type of material is of course much more expensive than the equipment for a current clamp, but has the advantage of providing an exact value of the power consumed.

The value of the phase shift $\varnothing$ is fixed and depends on the material supplied. It is of course possible to make a measurement of the phase shift value, and subsequently measure only the current values, and then make a quick calculation of the consumed power.

As the routers under study are powered by an alternative electrical source, the solution we selected is the one based on the use of a wattmeter that will directly provide the value of the power consumed.

We have chosen the Fluke wattmeter NORMA 4000 [14] as it can measure both voltages and currents. It includes a serial interface and a software named NormaView which is able to record the measurements made on a computer.

\section{The Experimental Platform}

The necessary equipment being selected, the problem then deals with getting the value of the voltage and current consumed by the router without affecting its integrity. To do this, we built a box that sits between the router and its power source. This box provides a connection entry for measuring the voltage and current consumed by the router and to provide them to the wattmeter.

All our experiments were done on a router belonging to the Laastexp experimental platform [15] of the LAAS-CNRS Laboratory, Toulouse, France. The routers of this platform are the Juniper M7i routers equipped with two Gigabit Ethernet interfaces. The first is a Gigabit Ethernet fixed interface card, and the second is a Gigabit Ethernet Physical Interface Card (PIC) with Small Form-factor Pluggable (SFP). Each of these interfaces is connected to a VLAN consisting of two computers. 


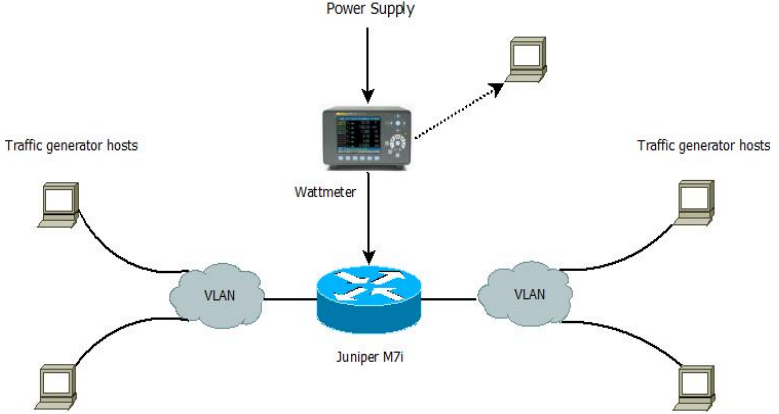

Fig. 1. Laboratory testbed

\section{EXPERIMENTS}

The goal with the experiments is to provide tangible results that can serve as a basis for giving a roadmap for future work in the field of green-networking. We do believe that for designing energy efficient protocols, the consumption characteristics of network devices must be established first.

For the experiments, the Jugi's Traffic Generator (JTG) has been used. This traffic generator can generate both UDP and TCP stream. It also allows the specification of the DSCP field (Differentiated Service Code Point). This possibility makes possible to test the effect of Quality of Service (and different queue management policies) on the power consumption.

Each of the following experiments took place over a period of 10 minutes with a gap between each measurement set to 250 milliseconds. The traffic generator generates UDP flows consisting of packets of 1500 bytes (1500 bytes being the Maximum transmission Unit allowed - MTU).

Note that the power consumed by the router when it is inactive is $92.17 \mathrm{~W}$.

\section{A. Impact Of Network Load}

To start, the first measurement series addresses the power consumed when the value of the bandwidth to be computed by the router increases. The range of traffic goes from $100 \mathrm{Mbps}$ to $800 \mathrm{Mbps}$, each step corresponding to an increment of 100 Mbit / s.

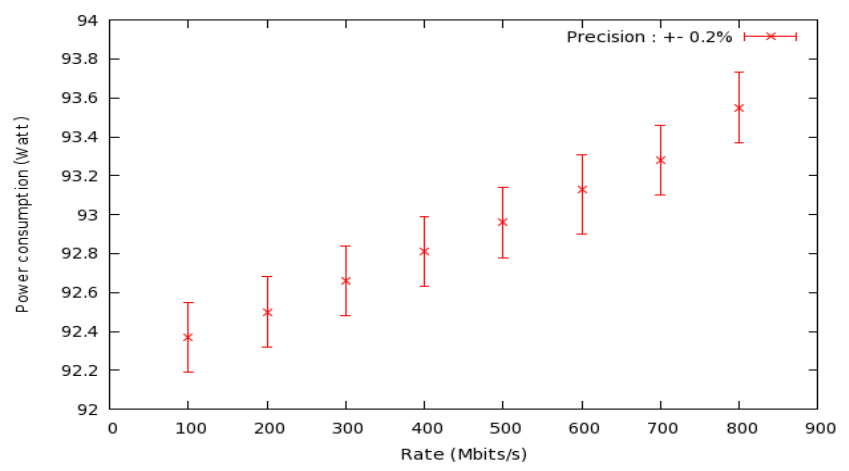

Fig. 2. Router electrical consumption for computing UDP traffic at different bit rates.

Figure 2 shows that when the load increases, the consumption of the router also increases. For a traffic load of $80 \%$ of the total bandwidth, an increase in the power consumption of $1.4 \mathrm{~W}$ has been observed. This increase corresponds to a variation in the power consumption of $1.55 \%$. These changes are still low and leave us to believe that our router is left of the category On/ Off: The variations in power consumption obtained when the level of traffic increases are small compared to the average power consumption of the router.

\section{B. Impact of Quality of Service (QoS) management}

For study the impact of QoS mechanisms of routers on their own consumption, the main active queue management strategies - Tail Drop, Randome Early Detection, (RED) and Weighted Fair Queuing (WFQ) - were tested. For Tail-Drop and RED, a single queue was set up. For testing the influence of WFQ, the traffic was dispatched between four queues (corresponding to four classes of traffic). It was also checked whether some differences arise if four, three or only two queues were used. The total bandwidth to be computed by the router was always kept the same in all presented experiments.

\begin{tabular}{|c|c|c|}
\hline AQM & $\begin{array}{c}\text { Number of active } \\
\text { queue }\end{array}$ & $\begin{array}{c}\text { Power } \\
\text { Consumption } \\
\text { (W) }\end{array}$ \\
\hline Tail Drop & 1 & 93.15 \\
\hline RED & 1 & 93.14 \\
\hline WFQ & 1 & 93.16 \\
\hline WFQ & 2 & 93.13 \\
\hline WFQ & 3 & 93.18 \\
\hline WFQ & 4 & 93.2 \\
\hline
\end{tabular}

Table 1. Electrical consumption for different QoS strategies. The bit rate of the total traffic equals $526 \mathrm{Mbits} / \mathrm{s}$.

Table 1shows that whatever the QoS and related queuing strategy, it is impossible to exhibit any difference on the power consumed by the router. This experience however allowed us to perform another test that has brought results quite surprising. Indeed to test the influence of the QoS on the power consumption it is necessary to change the software configuration. But as we shall see in the next section theses changes have a significant impact.

\section{Router Consumption while Loading new Router Configurations}




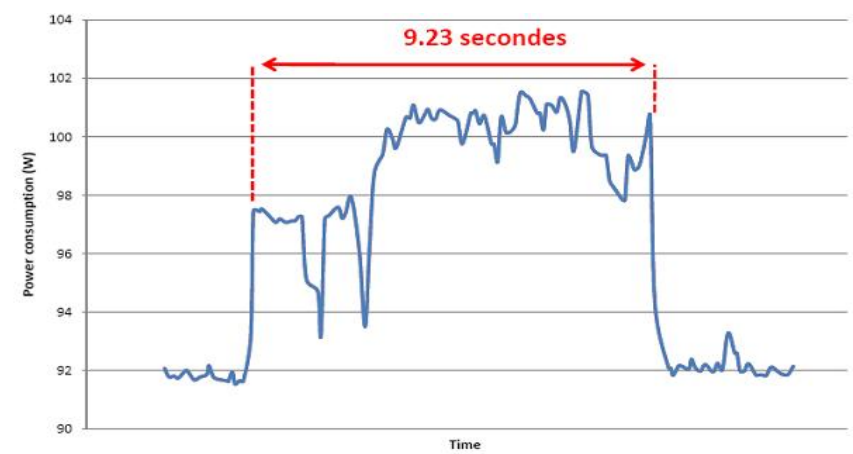

Fig. 3. Energy consumption when the configuration is loaded using a script shell.

The first experiment dealt with measuring the energy consumption and related changes when a new configuration is loaded and installed on the router by mean of a script shell. This choice was first made for the sake of saving our working time. Results are depicted on Figure 3. The figure especially shows that the consumption is the higher that was observed during all the performed experiments. The duration of this consumption increase is rather short but cannot be ignored anyway. It can be of significant importance for driving future work on how new management protocols should be designed. The router consumption in such experiment can be decomposed in several parts. However to get into such details, it was necessary to move from an automatic loading of new router configuration (by mean of a shell script) to a manual process for separating the different loading and installing phases. The results are depicted on Figure 4.

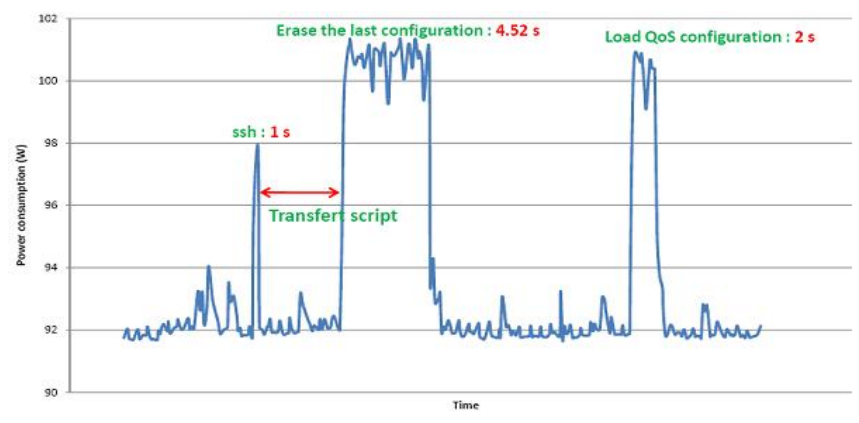

Fig. 4. Energy consumption when the router configuration is loaded manually and step by step.

Figure 4 shows that the electrical consumption consists of: - the launch of the ssh connection,

- the loading by removing the old configuration,

- and the addition of new QoS configuration to the original configuration.

Note that during the transfer phase of the configuration file itself, no consumption increase is observed. The complete set of results nevertheless shows that it would be preferable not to reconfigure hardware too often.

\section{BGP}

Moreover, this result led us to perform a final experiment for examining the influence of an update of the routing table due to a BGP announcement (a kind of configuration update which arise frequently for a router when used in the Internet). For this purpose, another router of the Laasnetexp platform [15] was used. This router is also a Juniper M7i with exactly the same hardware configuration as the one used in previous experiments.

When the establishment of the session was operational, a new static route was added to the auxiliary router. It was then possible to observe whether the update of the routing table on the first router has an influence on the power consumption. Results obtained clearly exhibited that BGP updates do not contribute to any increase of the power consumption.

\section{E. Rebooting the Router}

This section addresses measurements of the time needed for a router to start, and the related energy consumption.

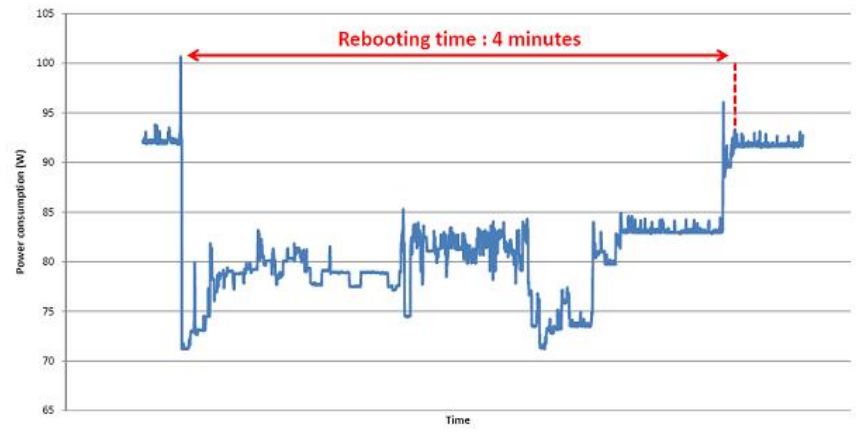

Fig. 5. Energy profile when the router is rebooting.

Figure 5 shows that this router restart procedure takes 4 minutes and exhibits the different consumption rates. During this restart period, the router is of course not available. This can for example illustrate the energy cost of possible attacks on routers.

\section{F. Ongoing experiments}

Finally, we would like to conclude this part on experiments by drawing your attention to a very interesting result that we have obtained but for which we do not have yet any explanation. Regularly we get energy consumption increases following a regular pattern: several successive peaks of power consumption. They appear in average 52 times per month. 


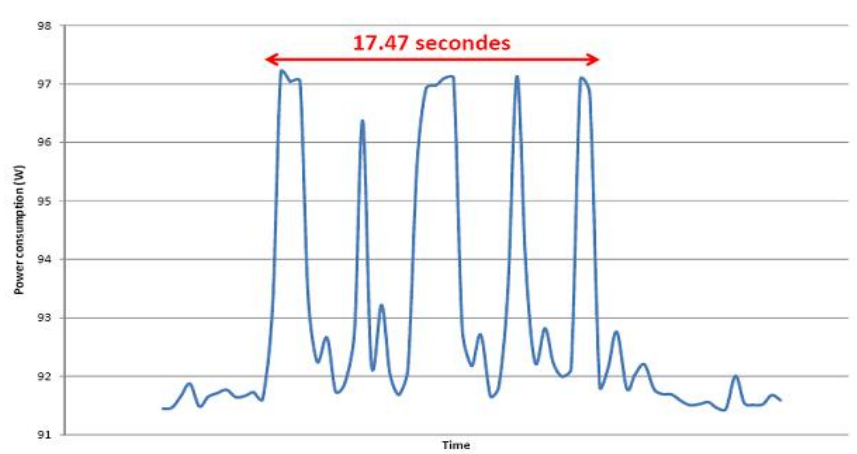

Fig. 6. Still unexplained energy consumption patterns.

Figure 6 shows that the magnitude of this energy profile is similar to that obtained when the configuration is loaded. Up to now, the origin of this phenomenon is unknown. One way we will follow consists in setting DAG probe in the network to determine whether the sudden increase of power consumption during "inactive" periods of the router is due to traffic characteristics or is due to internal computing of the router.

\section{G. Discussion}

After analyzing these first experimental results, it comes out that they were a bit surprising. If it was initially not surprising that traffic increases lead to increases of router power consumption, the fact that QoS enforcement strategies at the level of queues does not affect the power consumption of the router is surprising and at the opposite of what we thought. It then let think that line card computing does not cost much in terms of energy, while router switch fabric does. This however opens a way of thinking future researches for energy saving techniques in next generation routers. In addition, given these results and taking as reference the energy profiles issued by [12], it is possible to classify our routers as On/Off equipment category. Using such result for establishing a standard approach to energy aware routing profile could be interesting but not among the most relevant. We indeed would like to draw attention to the proxying technique.

As we have seen the router's startup time is very long. The technique of proxying requires shifting the management of asleep network equipments to an entity that will save all the packets and respond to network requests until the restart of the asleep equipment. But for routers expecting a time of several minutes for restarting them is very consistent. Such technique could then be implemented only when facing a network in which many paths are always available.

We could also imagine, during periods of low network load, to concentrate traffic on specific routes, turning off unused routers and gradually as the load increases rekindle the router off [11].

\section{DISCUSSION ON GREENING THE INTERNET}

The first measurement performed on power consumption of network equipments exhibited that:
- The consumed energy depends on the volume of computed traffic;

- The queue management policies have no impact on the router consumption;

- The equipment reconfiguration engenders overload power consumption, but BGP updates do not.

Related to the Internet design, management and evolution, such results mean:

- Whatever the kind of QoS management planned to be integrated in the network (class based as DiffServ, extensions of BGP for QoS as QBGP, ...), its impact on the electrical power consumption would be transparent. This is definitely not a direction to follow for greening the Internet, and research on QoS management can continue its path without considering the green dimension.

- The electrical power consumed by a router is very high, even if no traffic is forwarded by the router. If the router is fully loaded, the increase in the electrical power consumption is less than $2 \%$ of the electrical power consumed by an "empty" router (around 92 Watts in our experiments for an empty router, rising up to almost 94 Watts when fully loaded). The increase in the amount of traffic transferred on the Internet is a dimension to consider for designing a greener Internet. Internet traffic engineering is definitely the key problem to address, as for economical reasons, it would be impossible to force users to reduce the amount of data they send on the Internet.

- Network management must definitely limit the changes of router configuration. In any case, router configuration changes are very infrequent and unusual as they arise only when the routing, security or forwarding policies have to be changed (which are major strategic changes arising generally on a yearly basis), or when a bug is detected in the configuration of one or of a set of routers. This high consumption of electrical power for changing router configuration is then not a limitation. The good news is nevertheless that a route change advertized by BGP does not cost anything in terms of electrical power consumption. It lets open many possibilities for new traffic engineering strategies and policies.

Given the knowledge we currently have on the Internet topology and Internet traffic characteristics, a very simple solution puts one step out of the pack. Indeed, during nights, week-ends and days off, it has been widely observed a decrease of $80 \%$ of the Internet traffic. This decrease has been observed on all the ASes of the Internet, an AS being the atomic management unit of the Internet. It corresponds to a network domain under a single management and decision entity - generally the network operator. The internet consists nowadays of around 15,000 of theses ASes, individually and autonomously designed and managed. In addition, except 3 or 4 of these operators which are providing intercontinental interconnections, they all are concentrated on a limited geographic area - in general at the scale of a country or a state - meaning that it is night at the same time for all the routers of the related AS. Second, the topology of the Internet is of the "small world" type [16] meaning that nodes in this topology 
have a large degree (a high number of interconnection with other routers), or that, at least, any router of the Internet can be reached in few hops.

Relying on our electrical consumption results and the known characteristics on Internet traffic and topology, it is obvious that one very simple, very fast, and very easy to implement solution would be to switch off most of the routers of the ASes during nights, week-ends, days off, and, why not, vacation periods. Given the "small world" characteristic of the topology, the network is meshed enough to have fast and efficient connections between all border routers in the AS, even if most of the core routers are switched off. Given that the traffic is reduced by $80 \%$ in average, and that bandwidth of network is still largely overprovisioned, it is obviously possible to find a new topology with only $20 \%$ of the core routers (this is a property of "small world" networks which keep their characteristic even if some nodes are removed). The $2 \%$ increase of router electrical power consumption when forwarding 5 times more traffic than usually at the same period (the load of the router would, whatever happens, remain lower than 30 or $40 \%$ of its total capacity) is largely compensated by the fact that $80 \%$ of the routers would be switched off for 12 hours (night), 1 day (day off) or 2 days (week-end)... Even if the electrical power consumption will be increased by $2 \%$ for few minutes when restarting the routers, the global gain is significant. And the advertizing of new routes of the AS before shutting down or right after switching on the routers has no energy cost.

The demonstration of this statement is obvious given our measurement results on electrical power consumption. However, it is only possible to practically simulate it by generating artificial "small world" topologies. Given that the network topology is very strategic for network operators, and that this information is kept secret, it is quite impossible to assess our energy consumption reduction strategy on real AS topologies (the only freely available topologies are out of date).

Finally, this easy and fast solution relying on switching off routers when they are not absolutely needed for efficiently forwarding traffic is not generally considered in the literature; it generally addresses cases for which all nodes have mandatorily to stay up. Based on our knowledge about the Internet and its traffic, we argue that this not the case for the Internet, and that during periods of low activity many routers can be switched off without performance decrease. The solution is fast, simple, straightforward, easy, and can be implemented at almost no cost. We also argue it is more efficient, less time consuming, and less costly than working on re-designing network mechanisms and protocols.

\section{CONCLUSION}

In this paper, we first addressed the measurement of the power consumption of network equipments, specifically taking into account measurement accuracy and transparency. The first measurement performed addressed router energy consumption and exhibited that:

- The consumed energy depends on the volume of computed traffic;
- The queue management policies have no impact on the router consumption;

- The equipment reconfiguration engenders overload power consumption, but BGP updates do not.

Finally, despite this measurement work on router consumption is at its early stage, obtained results indicate good opportunity for the future in greening communications. Therefore, a discussion on possibilities for greening the Internet - or at least its ASes which are the atomic part of the Internet on which network operators can act - was started. Despite no concrete evaluation work was conducted yet on such ASes, it is obvious that significant energy consumption gains can be performed in the Internet; during low activity periods, the gain is around $80 \%$. In addition, the current topology of the Internet permits such gains. This work will of course continue to get deeper into the details of router energy consumption for continuing reducing energy wasted in the global Internet.

\section{REFERENCES}

[1] “Where Does Power Go?”, http://www.greendataproject.org/, January 2008

[2] H. Breuil, D. Burette, B. Flüry-Hérard, J. Cueugniet, D. Vignolles, H. Boisson, "Rapport - TIC et développement durable", France, Department of Ecology, December, 2008

[3] M. Gupta, S. Singh "Greening of the Internet", Proceedings of ACM SIGCOMM, Karlsruhe, Germany, August 2003

[4] S. Nedevschi, L. Popa, G. Iannaccone, S. Ratnasamy, D. Wetherall ,"Reducing Network Energy Consumption via Sleeping and RateAdaptation", $5^{\text {th }}$ Symposium on Networked Systems Design and Implementation, San Francisco, California, U.S.A, Apr. 16-18, 2008

[5] B. Nordman, K. Christensen, "Reducing the Energy Consumption of Networked Devices”, IEEE 802.3 tutorial, San Francisco, California, U.S.A, July 19, 2005

[6] M. Bennett, K. Christensen, B. Nordman, Ethernet Alliance "Improving the Energy Efficiency of Ethernet : Adaptive Link Rate Proposal”, Ethernet Alliance, Version 1.0, July 15, 2006

[7] B. Nordman, K. Christensen, "Improving the Energy Efficiency of Ethernet-Connected : A proposal for Proxying”, Ethernet Alliance, Version 1.0, September, 2007

[8] L. Chiaraviglio, D. Ciullo, E. Leonardi, M. Mellia, "How mutch Can The Internet Be Greened?”, IEEE GLOBECOM Workshops, 2009

[9] V. Soteriou, L. Peh, "Dynamic Power Management for Power Optimization of Interconnection Networks Using On/Off Links”, Proceedings of the 11 th Symposium on High Performance Interconnects, IEEE COMPUTER SOCIETY, 2003

[10] M. Gupta, S. Singh, "Dynamic Ethernet Link Shutdown for Energy Conservation on Ethernet Links”, ICC, IEEE International Conference, 2007

[11] L. Chiaraviglio, M. Mellia, F. Neri, "Reducing Power Consumption in Backbone Networks”, ICC, IEEE International Conference, 2009

[12] J. Cardona Restrepo, C. Gruber, C. Mas Machuca, "Energy profile Aware Routing”, ICC, IEEE International Conference, 2009

[13] J. Chabarek, J. Sommers, P. Barford, C. Estant, D. Tsiang, S. Wright, "Power Awareness in Network Design and Routing”, IEEE INFOCOM 2008, Phoenix, USA, April 2008

[14] “Fluke electronics”, http://www.fluke.com/, 2010

[15] http://homepages.laas.fr/owe/laasnetexp.fr/laasnetexp.fr.htm

[16] M. Faloutsos, P. Faloutsos, C. Faloutsos, “On Power-Law Relationships of the Internet Topology”, ACM SIGCOMM conference, Boston, USA, 1999 